\title{
Habituation of Rostral Anterior Cingulate Cortex to Repeated Emotionally Salient Pictures
}

\author{
K Luan Phan*,', Israel Liberzon ${ }^{1,4}$, Robert C Welsh², Jennifer C Britton ${ }^{3}$, Stephan F Taylor \\ 'Department of Psychiatry, University of Michigan, Ann Arbor, Ann Arbor, MI, USA; ²Department of Radiology, University of Michigan, Ann Arbor, \\ Ann Arbor, MI, USA; ${ }^{3}$ Neuroscience Program, University of Michigan, Ann Arbor, Ann Arbor, MI, USA; ${ }^{4}$ Psychiatry Service, Ann Arbor VAMC, Ann \\ Arbor, MI, USA
}

\begin{abstract}
Habituation of the neural response to repeated stimuli has been well demonstrated for subcortical limbic regions responding to emotionally salient stimuli. Although the rostral or affective division of the anterior cingulate cortex ( $r A C C$ ) is also engaged during emotional processing, little is known about the temporal dynamics of this region in sustained evaluation of emotional salience. Using a test/retest design, the present study assessed habituation in the human brain with functional magnetic resonance imaging. Eight healthy subjects were exposed to two repeated runs of aversive, neutral, and blank images. Activation of the rACC to negatively valenced pictures occurred only in the first session, and this activation was significantly greater in the first relative to the second session. Additionally, medial prefrontal cortex, hippocampal, and amygdalar activations were noted during the first, but not second, presentation of aversive pictures. These findings highlight the phasic activity of the rACC in emotional processing consistent with habituation. Neuropsychopharmacology (2003) 28, I344-1350, advance online publication, 23 April 2003; doi: I0. I038/sj.npp. I 300 I86
\end{abstract}

Keywords: functional magnetic resonance imaging; prefrontal cortex; emotion; attention; pictures; habituation

\section{INTRODUCTION}

Habituation of a neural response to repeated exposure to sensory stimuli is a well-documented phenomenon (Siddle, 1991; Sokolov, 1963; Thompson and Spencer, 1966), particularly during emotional processing. Specifically, several groups have demonstrated that the amygdala rapidly habituates to repeated presentations of emotional faces (Breiter et al, 1996; Whalen et al, 1998b; Wright et al, 2001) and pictures (Fischer et al, 2000; Irwin et al, 1996). These studies suggest that activity in subcortical limbic regions (eg amygdala, hippocampus) may be modulated by repeated exposure. However, the temporal dynamics of other limbic, as well as paralimbic and cortical, areas that are also implicated in emotion processing remain unclear. Two recent studies demonstrate apparent habituation effects to affective faces in prefrontal regions (dorsolateral and medial prefrontal cortex (DLPFC and MPFC) and precentral gyrus), thought to play a role in the cognitive control of attention (Feinstein et al, 2002; Wright et al, 2001). Another important

* Correspondence: Dr KL Phan, Department of Psychiatry, University of Michigan, UH9D, University Hospital, Box 0II8, I500 E. Medical Center Dr, Ann Arbor, MI 48 I09-0 I I 8, USA, Tel: + I 7349369280 , Fax: + I 734936 7868, E-mail: luan@umich.edu

Received 20 November 2002; revised 05 February 2003; accepted 10 February 2003

Online publication: 26 February 2003 at http://www.acnp.org/citations/ Npp022603419/default.pdf candidate to participate in this process is the anterior cingulate cortex (ACC) which has been hypothesized to mediate the allocation of attentional resources to emotionally arousing stimuli (Niedenthal and Kitayama, 1994). Furthermore, the rostral or affective division of the anterior cingulate (rACC) has been implicated in the evaluation of emotional salience in concert with the amygdala and other limbic regions, although this finding is inconsistent (Bush et al, 2000; Devinsky et al, 1995; Phan et al, 2002; Whalen et al, 1998a). If present, habituation would effectively reduce the ability to detect a signal or activation in the ACC in paradigms utilizing repeated exposure, but no direct examination of the activity of this region with repeated stimulus presentations has been reported in the literature.

This study sought to examine the habituation of brain activity to repeated presentations of emotional pictures with general aversive content using functional magnetic resonance imaging (fMRI). Given the findings from the earlier work with emotional faces, we hypothesized that habituation of brain responses in the prefrontal cortex (MPFC and DLPFC), rACC, amygdala, and hippocampus could be demonstrated.

\section{RESULTS}

\section{Subjective Self-Reports}

All subjects tolerated exposure to the stimuli without difficulty. On-line ratings (mean \pm SD) of stimuli 
confirmed the intended manipulation of content on unpleasantness from blank $(1.00 \pm 0.01)$ to neutral $(1.08 \pm 0.10) \quad$ to aversive pictures $(2.79 \pm 0.13)$ $(\mathrm{F}(2,44)=1902.15 ; p=0.000)$. There were no significant effect of time (T1 vs T2) across all three stimulus types (F $(1,45)=0.01, p=0.91)$, or time by condition interaction $(\mathrm{F}(1,2)=0.48 ; p=0.62)$. Specifically, unpleasantness ratings for the aversive stimuli did not differ between the first $(2.77 \pm 0.11)$ and second $(2.80 \pm 0.15)$ presentations $(\mathrm{F}(1,1 \overline{4})=0.33 ; p=0.57)$.

\section{Functional MRI Results}

First presentation vs second presentation (T1-T2). Consistent with habituation, a significant decrement in blood level oxygen-dependent (BOLD) signal change was detected at the same focus within the rACC for both aversive (AV) - blank (BL) and aversive - neutral (NT) contrasts with repeated presentations (Table 1, Figure 1), but not at other a priori regions. The rACC decrement was present even with correction for multiple comparisons $(p<0.05$, corrected) at the cluster level across the entire brain. Extracted time course data of the BOLD signal response to aversive pictures at the $\mathrm{rACC}$ exhibits the decrement in signal over the $12(\mathrm{AV}-\mathrm{BL})$ blocks of the study (T1: Blocks 1-6; T2: Blocks 7-12). The generalizability of the rACC habituation is demonstrated with individual data from all eight subjects (Table 2). We found no differences with respect to gender. For completeness and to highlight some specificity for the rACC finding, we examined the data at a lower post hoc threshold of $p<0.01$ (uncorrected, exceeding five voxels) in order to present data for foci at other a priori regions (Table 1). However, we emphasize that these peaks did not reach $a$ priori statistical significance threshold. No a priori region exhibited significant BOLD decrease with repetition ( $\mathrm{T} 1$ vs $\mathrm{T} 2$ ) in the NT-BL comparison. These results remained true even after the significance threshold was reduced to $p<0.05$, uncorrected. For both the $(\mathrm{AV}-\mathrm{BL})_{\mathrm{T} 1}-(\mathrm{AV}-\mathrm{BL})_{\mathrm{T} 2}$ and the $(\mathrm{AV}-\mathrm{NT})_{\mathrm{T} 1}-(\mathrm{AV}-\mathrm{NT})_{\mathrm{T} 2}$ contrasts, no nonpredicted regions survived significance threshold ( $p<0.05$, corrected). Furthermore, sensory-motor cortices such as the premotor-motor cortex, occipital cortex, and cerebellum did not show habituation (Figure 2).
First presentation (T1). During T1, we found activation in the $\operatorname{rACC}$ for $\mathrm{AV}-\mathrm{BL}((k, \#$ of voxels $)=74,(3,39,15)$, $Z=4.45)$ and in a cluster that included the rACC and MPFC for $\operatorname{AV}-\mathrm{NT}((k)=61,(15,45,9), Z=4.55 ;(6,45,9)$, $Z=4.00 ;(9,51,15), Z=3.41)$. Other activations in a priori regions were noted during $\mathrm{T} 1$ in the $\mathrm{AV}-\mathrm{BL}$ contrast in bilateral hippocampus $((\mathrm{k})=23,(-21,-30,-6), Z=3.96$; $(\mathrm{k})=10,(21,-18,-12), Z=3.49), \operatorname{MPFC}((\mathrm{k})=13,(-9,63$, $30), Z=3.93)$, and left extended amygdala $((\mathrm{k})=7,(-9,0$, $-12), Z=3.27)$. Of note, by lowering the spatial extent threshold, activation of the left amygdala $((\mathrm{k})=2,(-21,-6$, $-15), Z=3.18 ;(\mathrm{k})=2,(-18,-3,-15), Z=3.35)$ was evident during $\mathrm{T} 1$ in both $\mathrm{AV}-\mathrm{BL}$ and $\mathrm{AV}-\mathrm{NT}$ contrasts, respectively. No rACC activation was noted in the NT-BL comparison.

Second presentation (T2). Unlike T1, during T2, no a priori region reached significance $(p<0.001$, uncorrected) in either the $\mathrm{AV}-\mathrm{BL}$ or $\mathrm{AV}-\mathrm{NT}$ contrasts; this remained true even after the significance threshold was leniently reduced to $p<0.05$, uncorrected. Specifically, during T2, we found no activation of the ACC, as well as an absence of any significant deactivation, confirming that the decrement in response from $\mathrm{T} 1$ to $\mathrm{T} 2$ represents a difference in activation, and not deactivation during T2 (Figure 1). No rACC activation was noted in the NT-BL comparison.

First and second $(T 1+T 2)$ presentation. When we combined $\mathrm{T} 1$ and $\mathrm{T} 2$ for $\mathrm{AV}-\mathrm{BL}$ and $\mathrm{AV}-\mathrm{NT}$ contrasts, none of the activations observed during $\mathrm{T} 1$, including the ACC, exceeded our significance threshold, even at an uncorrected $p<0.05$ (Phan et al, 2003). No rACC activation was noted in the NT-BL comparison.

\section{DISCUSSION}

The primary novel finding of this study involves the demonstration of habituation of activity of the ACC to repeated presentations of emotionally salient pictures. The anatomic location of the cluster with significant BOLD signal decrement falls within the rostral or 'pregenual' division of the ACC (Brodman Area 24a-c) (Drevets and Raichle, 1998; Mayberg, 1997), implicated in a number of

Table I fMRI Signal Decrement at A Priori Regions to Repeated Presentation of Emotionally Salient Pictures

\begin{tabular}{|c|c|c|c|c|c|}
\hline Contrast & Region & $(k)$ & $(x, y, z)$ & $\mathbf{Z}$ & $p$-value \\
\hline$(\mathrm{AV}-\mathrm{BL})_{\mathrm{T} 1}-(\mathrm{AV}-\mathrm{BL})_{\mathrm{T} 2}$ & $\begin{array}{l}\text { Anterior cingulate cortex }{ }^{a} \\
\text { Dorsolateral prefrontal cortex } \\
\text { Right hippocampus } \\
\text { Left hippocampus/amygdala }\end{array}$ & $\begin{array}{l}(|4|) \\
(55) \\
(33) \\
(15)\end{array}$ & $\begin{array}{l}(3,36,3) \\
(39,39,33) \\
(36,-15,6) \\
(-18,-18,-12)\end{array}$ & $\begin{array}{l}4.92^{b} \\
2.93 \\
2.83 \\
2.77\end{array}$ & $\begin{array}{l}0.000 \\
0.002 \\
0.002 \\
0.003\end{array}$ \\
\hline$(\mathrm{AV}-\mathrm{NT})_{\mathrm{T} 1}-(\mathrm{AV}-\mathrm{NT})_{\mathrm{T} 2}$ & $\begin{array}{l}\text { Anterior cingulate cortex }{ }^{\mathrm{a}} \\
\text { Right hippocampus } \\
\text { Anterior medial prefrontal cortex }\end{array}$ & $\begin{array}{l}(30) \\
(19) \\
(14)\end{array}$ & $\begin{array}{l}(3,36,3) \\
(33,-24,-3) \\
(0,66,-3)\end{array}$ & $\begin{array}{l}3.93 \\
2.89 \\
2.69\end{array}$ & $\begin{array}{l}0.000 \\
0.002 \\
0.004\end{array}$ \\
\hline
\end{tabular}

a Significant $\mathrm{PMRI}$ signal decrement, threshold set at $p<0.00 \mathrm{I}$, uncorrected for multiple comparisons.

${ }^{b}$ Cluster-level significant at $p<0.05$, corrected for multiple comparisons across the entire brain.

'Significant $\mathrm{fMRI}$ signal decrement after threshold lowered to $p<0.0 \mathrm{I}$, uncorrected (italicized). For each maximal activation foci, cluster size ( $k$ ) in voxels, coordinates, $Z$ scores, and $p$-values are given. Coordinates are defined in MNI stereotactic space (Montreal Neurologic Institute) in millimeters: $x>0$ is right of the midsagittal plane, $y>0$ is anterior to the anterior commissure, and $z>0$ is superior to the anterior commissure-posterior commissure plane. $A V=a v e r s i v e, N T=$ neutral, $B L=b l a n k$;

$\mathrm{TI}=$ first presentation, $\mathrm{T} 2=$ second presentation. 


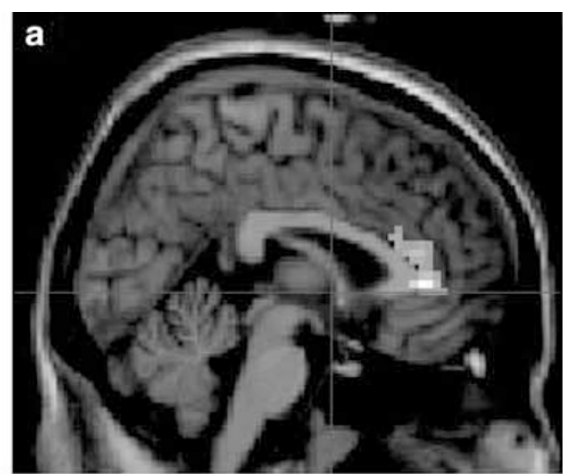

b $\quad f R O I$ of ACC
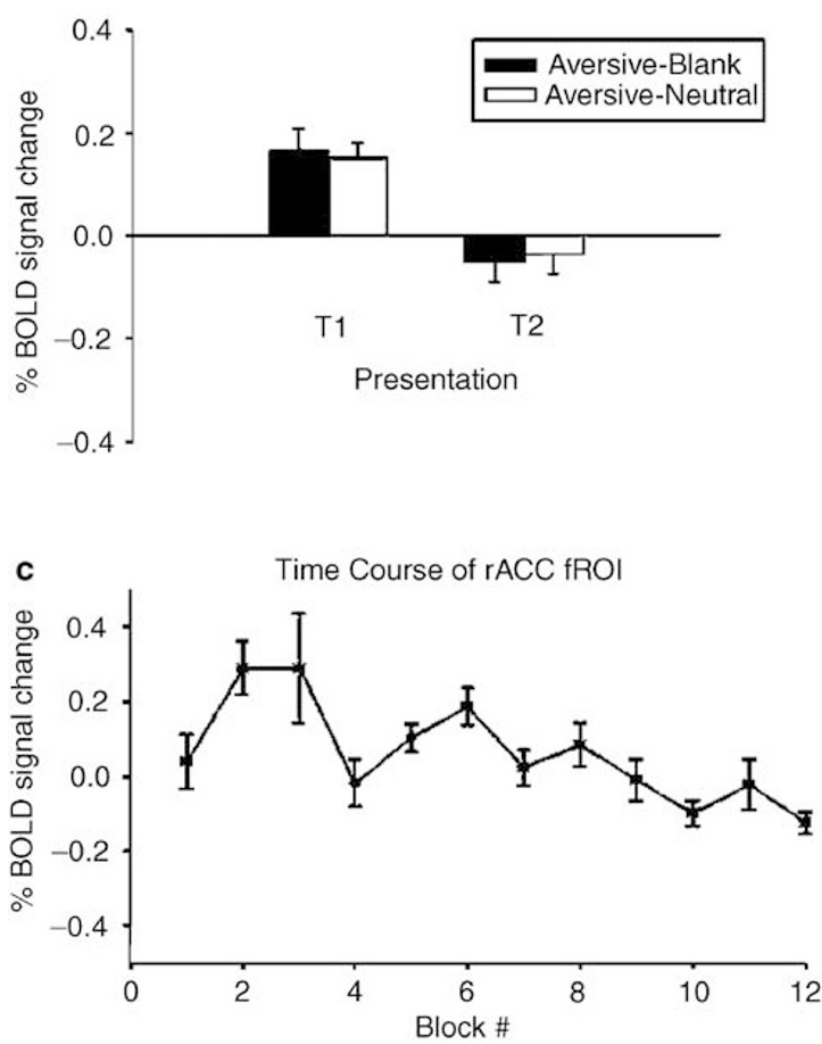

Figure I Habituation of the rACC. (a) SPM activation map showing significant decrement in $\mathrm{AMRI} B O L D$ response to repeated presentation of aversive stimuli relative to blank control condition (first presentation $(\mathrm{TI})$ vs second presentation (T2)) at the ACC $((k)=|4|$; $(3,36,3) ; Z=4.92$, $p<0.05$, corrected) displayed on $\mathrm{MNI}$ sagittal brain rendering. (b) Bar plot of $(A V-B L)$ and $(A V-N T)$ contrasts showing a significant decrease in percent BOLD signal change within an $\mathrm{AROI}$ of the ACC to aversive pictures as a function of repeated exposure. (c) Extracted time course of BOLD signal from rACC across 12 ( $\mathrm{AV}-\mathrm{BL}$ ) blocks of the study ( $\mathrm{TI}$ : Blocks I-6; T2: Blocks 7-12).

emotional operations including assessment of salience of emotional and motivational information and the regulation of emotional responses (Bush et al, 2000; Devinsky et al, 1995; Drevets and Raichle, 1998; Lane et al, 1997; Phan et al, 2002; Vogt et al, 1992; Whalen et al, 1998b). These findings extend earlier evidence of habituation of limbic subcortical (eg amygdala, hippocampus) and cortical (DLFPC, precentral gyrus) regions because of successive viewing of emotional faces and other salient visual stimuli (Breiter et al,
Table 2 Individualized Results of Habituation of the Rostral Anterior Cingulate Cortex (Brodman Area 24/32) ${ }^{a}$

\begin{tabular}{lllll}
\hline Contrast/subject \# & Gender & $(\mathbf{x}, \mathbf{y}, \mathbf{z})$ & $\mathbf{Z}$ & $\mathbf{p}$-value \\
\hline$(\mathrm{AV}-\mathrm{BL})_{\mathrm{T} I}-(\mathrm{AV}-\mathrm{BL})_{\mathrm{T} 2}$ & & & & \\
1 & Female & $(6,39,-12)$ & $5.4 \mathrm{I}^{\mathrm{b}}$ & 0.000 \\
2 & Male & $(3,15,6)$ & 3.62 & 0.000 \\
3 & Female & $(3,24,15)$ & $5.07^{\mathrm{b}}$ & 0.000 \\
4 & Female & $(0,42,0)$ & $4.92^{\mathrm{b}}$ & 0.000 \\
5 & Male & $(0,36,0)$ & 3.98 & 0.000 \\
6 & Female & $(0,33,6)$ & 3.73 & 0.000 \\
7 & Female & $(9,36,15)$ & 3.29 & 0.001 \\
8 & Male & $(0,18,15)$ & 3.40 & 0.001 \\
& & & & \\
$(\mathrm{AV}-\mathrm{NT})_{\mathrm{T} 1}-(\mathrm{AV}-\mathrm{NT})_{\mathrm{T} 2}$ & & & \\
1 & Female & $(0,42,-18)$ & $6.39^{\mathrm{b}}$ & 0.000 \\
2 & Male & $(-9,24,3)$ & 3.73 & 0.000 \\
3 & Female & $(3,42,0)$ & 4.10 & 0.000 \\
4 & Female & $(0,42,0)$ & 4.10 & 0.000 \\
5 & Male & $(-6,42,-12)$ & 3.53 & 0.000 \\
6 & Female & $(-3,45,33)$ & 3.84 & 0.000 \\
7 & Female & $(0,33,3)$ & 2.94 & 0.002 \\
8 & Male & $(0,33,6)$ & 1.98 & 0.024 \\
\hline
\end{tabular}

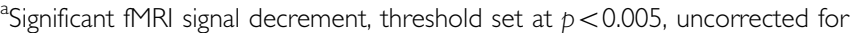
multiple comparisons, except for the $(\mathrm{AV}-\mathrm{NT})_{\mathrm{T} 1}-(\mathrm{AV}-\mathrm{NT})_{\mathrm{T} 2}$ contrast for subject 8 (italicized), threshold at $p<0.05$, uncorrected.

b Voxel-level significant at $p<0.05$, corrected for multiple comparisons across the entire brain. For each maximal activation foci at the rACC, MNI coordinates, $Z$ scores, and p-values are given. Abbreviations are described in Table I I.

1996; Feinstein et al, 2002; Fischer et al, 2000; Irwin et al, 1996; Whalen et al, 1998b; Wright et al, 2001).

Interestingly, although the decrement in response (from T1 to T2) did not exceed our predetermined statistical threshold, we did find significant activations in hippocampus, extended amygdala, and amygdala during the first, but not second, presentation of aversive pictures suggesting that some habituation also occur in these regions as expected. Therefore, habituation may not be isolated to the rACC, and other a priori regions (eg amygdala) expected to habituate also showed decrements in response that were not detectable only because of our significance threshold. Alternatively, several explanations could account for these results. First, the overall effect size of activation, and accordingly, the effect size of habituation in these regions to emotional pictures, may be smaller relative to emotional faces (Hariri et al, 2002; Phan et al, 2002). Second, our small sample size may have lowered the power to detect more subtle activation differences when $\mathrm{T} 1$ and $\mathrm{T} 2$ are compared directly. Third, these limbic regions may show more rapid habituation within $\mathrm{T} 1$, and thereby less magnitude of activation (Breiter et al, 1996; Wright et al, 2001). Also, individual differences in responses may add variability in limbic activity (Canli et al, 2001; Phan et al, 2003). Finally, if reciprocal interactions between rACC and amygdala exist (Liberzon et al, 2002; Morgan and LeDoux, 1999), then as rACC habituates during T2, and there is less inhibitory control, activity in amygdala may consequently increase. These possibilities would yield less power to detect a difference in limbic regions from $\mathrm{T} 1$ to $\mathrm{T} 2$, and/or to detect their activation overall.

Similar to habituation observed with negative valence facial expressions (Breiter et al, 1996; Feinstein et al, 2002; 

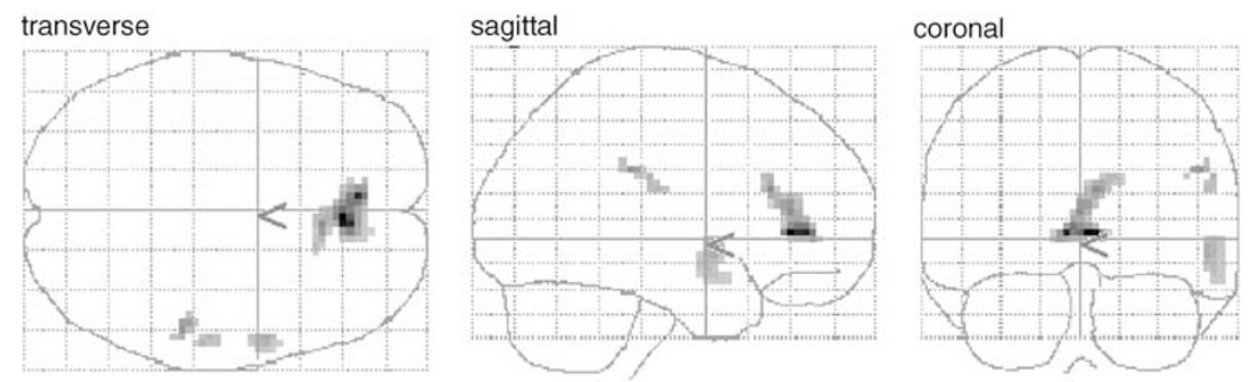

Figure 2 Absence of habituation in brain areas expected not to habituate ('control regions'). Displayed are three views (transverse, sagittal, and coronal) of the whole-brain ('glass brain') SPM activation map of the (AV-BL) $)_{\mathrm{T}}-(\mathrm{AV}-\mathrm{BL})_{\mathrm{T} 2}$ contrast, threshold at $p<0.00 \mathrm{I}$ (spatial extent $>$ five voxels). Note the absence of habituation (decrement in BOLD signal) throughout visual, sensory, and motor cortex.

Wright et al, 2001), the decrement in rACC activity with repeated exposure was specific to aversive pictures (eg decreases in rACC response to neutral pictures from T1 to T2 were not detected), relative to baseline (blanks) and control (neutral pictures) conditions. This is consistent with earlier work demonstrating that the prefrontal cortex, amygdala, and hippocampus habituate as the salience of the stimuli decreases with repetition (Breiter et al, 1996; Feinstein et al, 2002; Fischer et al, 2000; Whalen et al, 1998b; Wright et al, 2001). In addition to these regions, our findings suggest that the rACC also exhibits phasic temporal modulation of its activity by prior exposure, and that multiple interconnected brain regions (rACC, amygdala, hippocampus) undergo habituation during emotional processing. Interestingly, failure to detect any significant activation of the rACC (and other limbic regions) when both $\mathrm{T} 1$ and $\mathrm{T} 2$ sessions were combined demonstrates that not accounting for potential habituation in functional imaging paradigms that involve recurring exposure to salient stimuli could lead to false negative findings, leading to discrepant reports of activation patterns (Phan et al, 2002).

The repetition effect seen in rACC signal change could represent a number of processes including: (1) habituation to salient pictures over time, (2) habituation to repeated presentations of pictures with similar valence (eg aversive/ unpleasant), and/or (3) habituation to prior exposure of the same aversive picture (eg loss of novelty). If reciprocal interactions exist between rACC and limbic regions (Morgan and LeDoux, 1999), when the amygdala rapidly habituates during T1 (Breiter et al, 1996), cortical inhibitory control is no longer required and consequently there is less need to increase activity in rACC during T2. Additionally, the rACC may be responding to inputs from limbic brain regions (eg amygdala) that habituate, effectively decreasing the 'demand' from the rACC to modulate this activity either directly or via other regulatory regions. For example, by habituating, the amygdala lowers its input to basal forebrain cholinergic system and the locus coeruleus noradrenergic system, which in turn modulates their input to regions such as the anterior cingulate (Aston-Jones et al, 1996; Holland and Gallagher, 1999; LeDoux 2000). The repetition effect could alternatively be explained by nonspecific reductions in task engagement, scanner drift, or changes in subject fatigue or perception, rather than to stimulus content. However, we found that activity in regions important for sensory and motor processing (eg premotor-motor cortex, visual cortex, and cerebellum) did not change from T1 to T2. This finding suggests that the habituation found in the rACC is not likely because of a generalized/whole-brain decreased response or because of systematic artifacts in data collection or analysis that would lead to a global phenomenon of reduced activity across the entire brain. Furthermore, a repetition effect of the rACC was not seen in the NT-BL comparison suggesting some specificity towards aversive/negatively valence stimuli. Also, our use of an automated image registration algorithm, conservative random effects analysis, contrasts in which 'baseline' and control conditions were subtracted within $\mathrm{T} 1$ and $\mathrm{T} 2$, and counterbalanced block order presentation would compensate for several of these potential confounds.

Habituation specific to the rostral ACC (eg without subcortical/limbic input) may have its own functional significance during emotional processing. The rostral or pregenual region of the ACC (Brodman Area 24a-c, 32, 33) is distinctively referred to as the 'affective division' (Bush et al, 2000; Vogt et al, 1992), and is primarily involved in assessing emotional salience and motivational information. Pertinent to this study, the rACC has also been linked to the mediation of emotional arousal (Critchley et al, 2000, 2001, 2002). As a marker of salience, emotional arousal determines the allocation of brain resources and heightens sensitivity to environmental cues (Lane et al, 1998, 1999; Niedenthal and Kitayama, 1994). The rostral ACC also appears important in self-awareness and self-referential appraisals while viewing affective pictures (Lane et al, 1997; Lane et al, 1998; Taylor et al, 2002), and during the internal generation of emotions (eg emotional recall of biographical events) (Phan et al, 2002). In this study, as the emotional value of a stimulus drops upon repeated presentations, there is less need for internal evaluation and responding, and therefore, activity in the rACC drops. Thus, we hypothesize that as subjective arousal (eg salience) diminishes from first to second presentation of aversive stimuli, rACC activity appropriately decreases. We did not find a convergence between subjective ratings of valence (eg a decrease in experienced unpleasantness from $\mathrm{T} 1$ to $\mathrm{T} 2$ ) and BOLD activity. This may suggest that the emotional processing associated with rACC activity is somewhat orthogonal to that which is indexed by the unpleasantness judgments, and reflects subjective arousal or an orienting response, rather than valence-related processing. 
Accordingly, subjective arousal and valence ratings of emotional pictures are not directly correlated (Lang et al, 1998) and response of the medial wall of the frontal cortex (including the ACC) is associated with individual ratings of emotional arousal, not valence (Phan et al, 2003). Future fMRI studies should clarify rACC function by dissociating arousal and valence based on task and stimulus characteristics. Alternatively, the lack of convergence between ratings and BOLD activity may also reflect: (1) the narrow range of ratings of unpleasantness $(1,2$, or 3$)$ that was used in order to simplify the rating task during the scanning but effectively decreased the sensitivity for detecting differences in subjective experience that might exist between $\mathrm{T} 1$ and $\mathrm{T} 2$; and/or (2) increased probability of Type II errors given our small sample size.

Several limitations of this study are worth mentioning. First, the small sample size limits a more complete evaluation of habituation to emotional pictures at other relevant brain regions besides the rACC to determine if they too habituate, or alternatively, if any dissociations or interactions exist, specifically between the rACC and limbic regions (eg amygdala). Our findings prompt replication with a larger sample. Second, because the stimulus set did not contain pictures with positive/pleasant valence, we cannot comment on whether habituation of the ACC would also occur in response to repeated exposure of approachrelated material as observed previously with happy facial expressions (Feinstein et al, 2002; Wright et al, 2001). Third, our test/retest block-related design made it difficult to assess fully for either changes in activity within runs or recovery of activity (or reduction of habituation). Further work exploiting event-related designs that allow analysis of discrete time course information over a few seconds, as well as using other types of salient stimuli with random presentations, will be needed to clarify these important issues.

In summary, the present results demonstrate for the first time that the rACC shows phasic fluctuations to repeated exposure of aversive visual stimuli consistent with habituation. This study also underscores the importance of examining the temporal dynamics of brain activation in fMRI design and analysis in studies of emotional processing.

\section{MATERIALS AND METHODS}

\section{Subjects}

Eight healthy right-handed volunteers (five females; mean age $24.4 \pm 2$ years (range, 19-28)) participated in the fMRI study. Subjects had no history of head injury, learning disability, or psychiatric illness including substance abuse/ dependence, as verified by a structured clinical interview. All subjects gave written informed consent after explanation of the experimental protocol, as approved by the local Institutional Review Board.

\section{Task Design}

The stimulus set consisted of 30 gray-scale AV and 30 grayscale NT pictures taken from the International Affective Picture System (IAPS) (Lang et al, 1997), and five BL gray images with a centered fixation cross (Taylor et al, 2000). Aversive pictures generally depicted mutilated bodies, explosions, car accidents, angry faces, dead animals, etc., while neutral pictures contained people, benign scenes of buildings and cars, live animals, neutral faces, etc. Subjects viewed counterbalanced blocks of $30 \mathrm{~s}$, equally distributed consisting of either five pictures of the same valence type (AV or NT) or five BL images; each picture was presented for $5 \mathrm{~s}$ with a $1 \mathrm{~s}$ interstimulus interval. The BL blocks served as a low-level 'baseline' condition, and the NT blocks served as a 'control' control, matched for luminance, faces/ figures, and complexity. In the first half of the study, the subjects viewed 18 blocks of stimuli (first presentation/test, T1) and in the second half (separated by approximately $2 \mathrm{~min}$ ), each subject was immediately retested using the same task with the same 18 blocks (second presentation/ test, T2), thereby exposing them to the same stimuli twice. Stimuli were displayed through a shielded LCD panel mounted on a head coil. While viewing each image, subjects made a button press rating each picture on the level of unpleasantness $(1=$ none, $2=$ mild, and $3=$ strong $)$.

\section{MRI Acquisition}

MRI was performed on a $3.0 \mathrm{~T}$ GE Signa system (Milwaukee, WI) using a standard RF coil. To reduce susceptibility artifact, whole-brain functional scans were acquired using a two-shot T2*-weighted spiral-scan pulse sequence (Stenger et al, 2002) with a shortened TE (TE $=15 \mathrm{~ms}, \mathrm{TR}=3000 \mathrm{~ms}$, freq $=64$ frames, flip angle $=65^{\circ}, \mathrm{FOV}=20 \mathrm{~cm}, 28$ oblique axial $4 \mathrm{~mm}$ contiguous slices/TR approximately parallel to the AC-PC line). The functional images of the two-shot spiral acquisition method were visually examined to ensure that there was no substantial signal blowout and had more signal recovery (eg less artifact) at the medial temporal lobe (including the amygdala) and the orbitofrontal cortex in comparison with the conventional one-shot acquisition (Phan et al, 2001, 2003; Stenger et al, 2002). A highresolution $\mathrm{T} 1 \mathrm{scan}$ was acquired to provide precise anatomical localization (3D-SPGR, TR $=25 \mathrm{~ms}$, min TE, $\mathrm{FOV}=24 \mathrm{~cm}$, slice thk $=1.4 \mathrm{~cm}$ ). Coimages were reconstructed off-line, using the gridding approach into a $128 \times 128$ display matrix, with an effective spatial resolution of $3 \times 3 \times 3 \mathrm{~mm}^{3}$ voxels.

\section{Image and Statistical Analysis}

Image analysis of the BOLD signal was performed with SPM 99 (Wellcome Institute of Cognitive Neurology, London, UK). Reconstructed data were realigned (Woods et al, 1998), slice time corrected (Oppenheim and Schafer, 1989), spatially normalized, high-pass filtered, and smoothed with a Gaussian filter $(8 \mathrm{~mm}$ full-width at half-maximum (FWHM)) to minimize noise and residual differences in gyral anatomy (Friston et al, 1995; Poline et al, 1995). In a standard block-design analysis of task-dependent activation, a condition-specific 'boxcar' covariate was constructed representing the occurrence of each block type, convolved with canonical response hemodynamic function and entered into the general linear model (Friston et al, 1995). Contrast maps for each subject were entered into a secondlevel, random effects group analysis (Holmes and Friston, 
1998), which accounts for scan-to-scan and subject-tosubject variability, and for scan drift. Effects at each voxel were calculated using a $t$-statistic, producing a statistical image and $Z$ distribution to test for habituation using the following contrasts: $\quad(\mathrm{AV}-\mathrm{BL})_{\mathrm{T} 1}-(\mathrm{AV}-\mathrm{BL})_{\mathrm{T} 2}$, $(\mathrm{AV}-\mathrm{NT})_{\mathrm{T} 1}-(\mathrm{AV}-\mathrm{NT})_{\mathrm{T} 2}$, and $(\mathrm{NT}-\mathrm{BL})_{\mathrm{T} 1}-(\mathrm{NT}-\mathrm{BL})_{\mathrm{T} 2}$. In addition, we also tested for activation in each session: $(\mathrm{AV}-\mathrm{BL})_{\mathrm{T} 1}$ only and $(\mathrm{AV}-\mathrm{NT})_{\mathrm{T} 1 \text { only }}$ for the first presentation, and $(\mathrm{AV}-\mathrm{BL})_{\mathrm{T} 2}$ only and $(\mathrm{AV}-\mathrm{NT})_{\mathrm{T} 2}$ only for the second presentation. In a priori defined regions (ACC, MPFC, dorsal lateral prefrontal cortex, amygdala, and hippocampus) derived from prior findings, we accepted activation that exceeded a probability of $Z>3.09(p<0.001$, uncorrected) similar to prior fMRI studies of emotion and/ or habituation (Feinstein et al, 2002; Hamann and Mao 2002; Hariri et al, 2002; Phan et al, 2003; Winston et al, 2002). To minimize false positive activations, we only accepted activations with a spatial extent exceeding five contiguous voxels, based on our smoothing kernel of $8 \mathrm{~mm}$ FWHM. In order to determine the regional specificity of habituation, we also examined brain areas (sensory-motor cortex) that we would not expect to habituate (Feinstein et al, 2002; Fischer et al, 2000; Wright et al, 2001) such as the premotor/motor cortex, occipital cortex, and cerebellum serving as 'control regions' at the same threshold. For nonpredicted regions, we report activation foci exceeding a threshold of $Z>5.30 \quad(p<0.05$, corrected). Areas that showed significant habituation were used to define functionally derived regions of interest (fROI) in order to extract raw time course data and percentage BOLD signal change averaged across subjects from $\mathrm{T} 1$ to $\mathrm{T} 2$.

\section{ACKNOWLEDGEMENTS}

We thank Thomas Nichols, Douglas Noll, Laura Decker, Samir Khan, and Keith Sudheimer for their assistance in this study. This research was supported in part by the APIRE/Janssen Fellowship (KLP), NIMH K08 MH01258 (SFT), the Ann Arbor Veterans Administration Medical Center (IL), and the University of Michigan fMRI Laboratory.

\section{REFERENCES}

Aston-Jones G, Rajkowski J, Kubiak P, Valentino RJ, Shipley MT (1996). Role of the locus coeruleus in emotional activation. Prog Brain Res 107: 379-402.

Breiter HC, Etcoff NL, Whalen PJ, Kennedy WA, Rauch SL, Buckner RL et al (1996). Response and habituation of the human amygdala during visual processing of facial expression. Neuron 17: 875-887.

Bush G, Luu P, Posner MI (2000). Cognitive and emotional influences in anterior cingulate cortex. Trends Cogn Sci 4: 215-222.

Canli T, Zhao Z, Desmond JE, Kang E, Gross J, Gabrieli JD (2001). An fMRI study of personality influences on brain reactivity to emotional stimuli. Behav Neurosci 115: 33-42.

Critchley HD, Elliott R, Mathias CJ, Dolan RJ (2000). Neural activity relating to generation and representation of galvanic skin conductance responses: a functional magnetic resonance imaging study. $J$ Neurosci 20: 3033-3040.
Critchley HD, Mathias CJ, Dolan RJ (2001). Neural activity in the human brain relating to uncertainty and arousal during anticipation. Neuron 29: 537-545.

Critchley HD, Melmed RN, Featherstone E, Mathias CJ, Dolan RJ (2002). Volitional control of autonomic arousal: a functional magnetic resonance study. Neuroimage 16: 909-919.

Devinsky O, Morrell MJ, Vogt BA (1995). Contributions of anterior cingulate cortex to behaviour. Brain 118(Pt 1): 279-306.

Drevets W, Raichle M (1998). Reciprocal suppression of regional cerebral blood flow during emotion versus higher cognitive processes: implication for interactions between emotion and cognition. Cogn Emotion 12: 353-385.

Feinstein JS, Goldin PR, Stein MB, Brown GG, Paulus MP (2002). Habituation of attentional networks during emotion processing. Neuroreport 13: 1255-1258.

Fischer H, Furmark T, Wik G, Fredrikson M (2000). Brain representation of habituation to repeated complex visual stimulation studied with PET. Neuroreport 11: 123-126.

Friston KJ, Holmes AP, Poline JB, Grasby PJ, Williams SC, Frackowiak RS, Turner R (1995). Analysis of fMRI time-series revisited. Neuroimage 2: 45-53.

Hamann S, Mao H (2002). Positive and negative emotional verbal stimuli elicit activity in the left amygdala. Neuroreport 13: 15-19.

Hariri AR, Tessitore A, Mattay VS, Fera F, Weinberger DR (2002). The amygdala response to emotional stimuli: a comparison of faces and scenes. Neuroimage 17: 317-323.

Holland PC, Gallagher M (1999). Amygdala circuitry in attentional and representational processes. Trends Cogn Sci 3: 65-73.

Holmes A, Friston KJ (1998). Generalizability, random effects, and population inference. Neuroimage 7: S754.

Irwin W, Davidson RJ, Lowe MJ, Mock BJ, Sorenson JA, Turski PA (1996). Human amygdala activation detected with echo-planar functional magnetic resonance imaging. Neuroreport 7: 1765-1769.

Lane RD, Chua PM, Dolan RJ (1999). Common effects of emotional valence, arousal and attention on neural activation during visual processing of pictures. Neuropsychologia 37: 989-997.

Lane RD, Fink GR, Chau PM, Dolan RJ (1997). Neural activation during selective attention to subjective emotional responses. Neuroreport 8: 3969-3972.

Lane RD, Reiman EM, Axelrod B, Yun LS, Holmes A, Schwartz GE (1998). Neural correlates of levels of emotional awareness. Evidence of an interaction between emotion and attention in the anterior cingulate cortex. J Cogn Neurosci 10: 525-535.

Lang PJ, Bradley MM, Cuthbert BN (1997). International Affective Picture System (Hariri et al): Technical Manaual and Affective Ratings. Gainesville, FL: NIMH Center for the Study of Emotion and Attention, University of Florida.

Lang PJ, Bradley MM, Cuthbert BN (1998). Emotion, motivation, and anxiety: brain mechanisms and psychophysiology. Biol Psychiatry 44: 1248-1263.

LeDoux JE (2000). Emotion circuits in the brain. Annu Rev Neurosci 23: 155-184.

Liberzon I, Zubieta JK, Fig LM, Phan KL, Koeppe RA, Taylor SF (2002). mu-Opioid receptors and limbic responses to aversive emotional stimuli. Proc Natl Acad Sci USA 99: 7084-7089.

Mayberg HS (1997). Limbic-cortical dysregulation: a proposed model of depression. J Neuropsychiatry Clin Neurosci 9: 471-481.

Morgan MA, LeDoux JE (1999). Contribution of ventrolateral prefrontal cortex to the acquisition and extinction of conditioned fear in rats. Neurobiol Learn Mem 72: 244-251.

Niedenthal PM, Kitayama S (1994). The Heart's Eye-Emotional Influences in Perception and Attention. Academic Press: New York.

Oppenheim AV, Schafer RW (1989). Discrete-Time Signal Processing. Prentice-Hall: Englewood Cliffs, NJ.

Phan KL, Taylor SF, Decker LR, Welsh RC, Noll DC, Nichols TE et al (2001). Detecting amygdala activations for aversive pictures 
with fMRI using 2-shot acquisition. Annual Meeting of the American College of Neuropsychopharmacology Scientific Abstracts, pp 228.

Phan KL, Taylor SF, Welsh RC, Decker LR, Noll DC, Nichols TE et al (2003). Activation of the medial prefrontal cortex and extended amygdala by individual ratings of emotional arousal: a functional magnetic resonance imaging study. Biol Psychiatry 53: 211-215.

Phan KL, Wager T, Taylor SF, Liberzon I (2002). Functional neuroanatomy of emotion: a meta-analysis of emotion activation studies in PET and fMRI. Neuroimage 16: 331-348.

Poline JB, Worsley KJ, Holmes AP, Frackowiak RS, Friston KJ (1995). Estimating smoothness in statistical parametric maps: variability of $p$ values. J Comput Assist Tomogr 19: 788-796.

Siddle DA (1991). Orienting, habituation, and resource allocation: an associative analysis. Psychophysiology 28: 245-259.

Sokolov E (1963). Perception and the Conditioned Reflex. Pergamon Press: Oxford.

Stenger VA, Boada FE, Noll DC (2002). Multishot 3D slice-select tailored RF pulses for MRI. Magn Reson Med 48: 157-165.

Taylor SF, Liberzon I, Koeppe RA (2000). The effect of graded aversive stimuli on limbic and visual activation. Neuropsychologia 38: 1415-1425.

Taylor SF, Phan KL, Decker LR, Liberzon I (2003). Subjective rating of emotionally salient stimuli modulates neural activity. Neuroimage 18: 650-659.
Thompson RF, Spencer WA (1966). Habituation: a model phenomenon for the study of neuronal substrates of behavior. Psychol Rev 73: 16-43.

Vogt BA, Finch DM, Olson CR (1992). Functional heterogeneity in cingulate cortex: the anterior executive and posterior evaluative regions. Cereb Cortex 2: 435-443.

Whalen PJ, Bush G, McNally RJ, Wilhelm S, McInerney SC, Jenike MA et al (1998a). The emotional counting Stroop paradigm: a functional magnetic resonance imaging probe of the anterior cingulate affective division. Biol Psychiatry 44: 1219-1228.

Whalen PJ, Rauch SL, Etcoff NL, McInerney SC, Lee MB, Jenike MA (1998b). Masked presentations of emotional facial expressions modulate amygdala activity without explicit knowledge. J Neurosci 18: 411-418.

Winston JS, Strange BA, O'Doherty J, Dolan RJ (2002). Automatic and intentional brain responses during evaluation of trustworthiness of faces. Nat Neurosci 5: 277-283.

Woods RP, Grafton ST, Holmes CJ, Cherry SR, Mazziotta JC (1998). Automated image registration: I. General methods and intrasubject, intramodality validation. J Comput Assist Tomogr 22: $139-152$.

Wright CI, Fischer H, Whalen PJ, McInerney SC, Shin LM, Rauch SL (2001). Differential prefrontal cortex and amygdala habituation to repeatedly presented emotional stimuli. Neuroreport 12 379-383. 